\title{
Quasi-Particle density of states and Thouless conductance of disordered $d$-wave superconductors
}

\author{
Bodo Huckestein ${ }^{1}$ and Alexander Althand ${ }^{2}$ \\ ${ }^{1}$ Institut für theoretische Physik III, Ruhr-Universität Bochum, D-44780 Bochum, Germany \\ ${ }^{2}$ Institut für theoretische Physik, Universität zu Köln, D-50937 Köln, Germany
}

(Received October 26, 2018)

KEYWORDS: d-wave superconductor, disorder, density of states, localization, Thouless numbers

Disorder in electronic systems suppresses diffusion and can lead to localization. ${ }^{1)}$ The origin of this behavior is enhanced backscattering due to quantum interference. In addition, disorder leads to a broadening of the density of states (DoS). However, the localization properties of a disordered system are usually not discernible from a study of the DoS. It is necessary to calculate two-particle properties or consider the influence of boundary conditions on energy eigenvalues as in the study of Thouless numbers to obtain information about localization. Another feature of disordered systems is the independence of their localization properties from details of the disorder in the system. For example, the range of the disorder affects non-universal quantities like the mean-free path but does not influence whether or not the system shows localization at all.

Recently, new symmetry classes came into focus that do not conform to the standard expectations about disordered systems outlined above. ${ }^{2}$ As we will show in this contribution, details of disorder do matter for these systems and localization properties leave their mark on the DoS. In particular, we look at a system that presents an idealization of a disordered $d$-wave superconductor. In our study we neglect effects of self-consistency and concentrate on the $d$-wave symmetry and influence of different kinds of disorder.

We consider the lattice quasi-particle Hamiltonian

$$
H=\sum_{i j ; \sigma}\left(t_{i j}-\mu \delta_{i j}\right) c_{i \sigma}^{\dagger} c_{j \sigma}+\sum_{i j} \Delta_{i j} c_{i \uparrow}^{\dagger} c_{j \downarrow}^{\dagger}+\text { h.c. }
$$

with the hopping matrix elements $t_{i j}$, chemical potential $\mu$, and order parameter $\Delta_{i j}$. The sums run over points of a two-dimensional square lattice with unit spacing and the operators $c_{i \sigma}^{\dagger}$ create a spin- $1 / 2$ particle of spin $\sigma$ at site $i$. In the following, we take only into account on-site potentials and nearest-neighbor hopping, $t_{i j}=\epsilon_{i} \delta_{i j}+t \delta_{i, j \pm e_{k}}$, where $e_{k}$ is the unit vector in $k$ direction. For convenience we set $\mu=0$ (the half-filled band.) The order parameter $\Delta_{i j}=\Delta\left(\delta_{i, j \pm e_{x}}-\delta_{i, j \pm e_{y}}\right)$

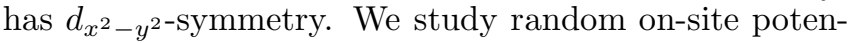
tial $\epsilon_{i}$ with a spatial correlation length $\xi{ }^{3)}$ The spectrum of (1) in the absence of disorder contains four low-energy sectors located near the wave-vectors $k=( \pm \pi / 2, \pm \pi / 2)$ (Dirac cones). Disorder couples the states of the unperturbed Hamiltonian but for long-ranged potentials $(\xi \gg 1)$ the coupling between the different Dirac cones

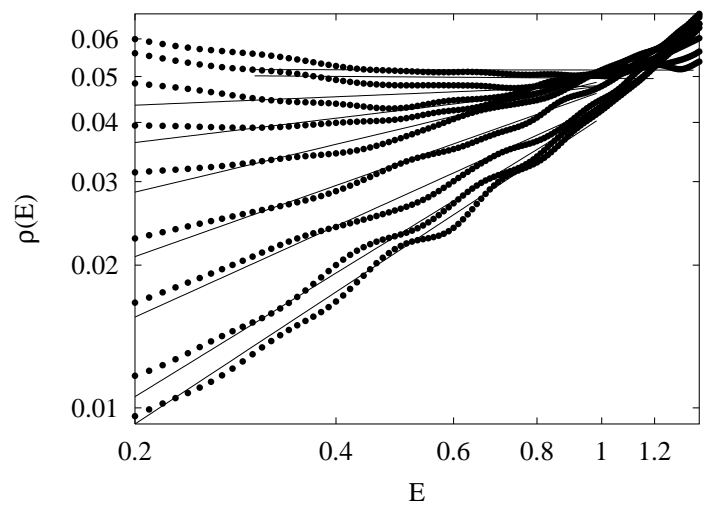

Fig. 1. Double logarithmic plot of the density of states for long-ranged disorder $(\xi=2)$. Disorder values are $W=$ $1,2, \ldots, 7,8,10$ (bottom to top). Dots $(\bullet)$ represent data and lines power law fits to the respective intervals.

will be very small and will only be relevant on very large length scales.

It has been predicted that for long-ranged disorder the spectrum of eq. (1) shows power law behavior with a non-universal exponent corresponding to a line of critical states: ${ }^{4,5)}$

$$
\rho(E) \sim|E|^{\alpha}, \quad \alpha=\frac{1-g}{1+g}, \quad g=\frac{W^{2}}{16 \pi t \Delta} .
$$

We indeed find numerically power law behavior for intermediate energies (Fig. 1) with the exponents well described by eq. (2) (Fig. 2). For larger values of the disorder, $g>1$, the DoS does not diverge but remains constant as a function of energy as has been argued by Gurarie. ${ }^{6)}$ In the nomenclature of Altland and Zirnbauer the case of long-ranged disorder conforms to the symmetry class $A \mathrm{III}^{2)}$

We now turn our attention to the case of short-ranged disorder, belonging to the symmetry class $C$ I. Here the DoS does not show power law scaling and is only weakly energy dependent for strong disorder. However, since the spectrum is particle-hole symmetric level repulsion of the eigenvalues near zero energy creates a microgap with a linear DoS, $\rho(E) \propto|E|$. The width of the microgap is given by the mean level spacing of a single localization volume $\Delta_{\xi}=\left(\rho_{0} \xi^{2}\right)^{-1}$ if the localization length $\xi$ is smaller than the system size $L$. Otherwise, it is given by the mean level spacing of the whole system. As a result, 


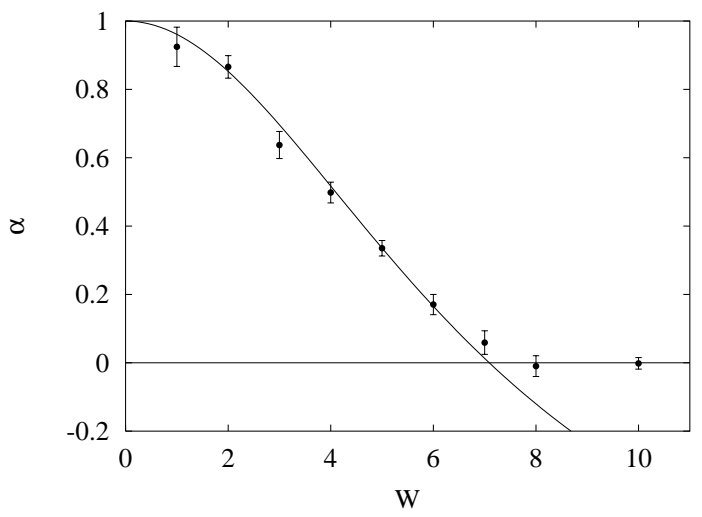

Fig. 2. Exponents $\alpha$ extracted from the fitted curves in Fig. 1 as a function of disorder $W$ for $\Delta=1$. The solid curve is the result eq. (2).

the width of the microgap first shrinks with increasing system size and finally saturates (see Fig. 3).

In an attempt to quantitatively analyze the crossover of the microgap width from mean level spacing of the whole system to that of a single localization volume, we have fitted the system size dependence of the slope $m$ of the microgap DoS to the form

$$
m(L)=m_{\infty} \frac{(L / \xi)^{2}}{1+(L / \xi)^{2}} .
$$

For small system sizes this expression is consistent with the system size dependence of the mean level spacing $\Delta \propto L^{-2}$ while it approaches a disorder dependent constant at large $L$. Fig. 4 shows that eq. (3) indeed provides a reasonable description of the crossover and that the localization length can indeed be deduced from the DoS.

Finally, we can check whether the crossover length extracted from the DoS corresponds to the localization length that governs transport. To this end we have averaged the typical Thouless numbers $g$ over a small energy interval near zero energy $(0<E<0.05$, less than a third of the width of the microgap). Fig. 5 compares the system size dependence of this quantity with an exponential decay with the crossover length obtained from

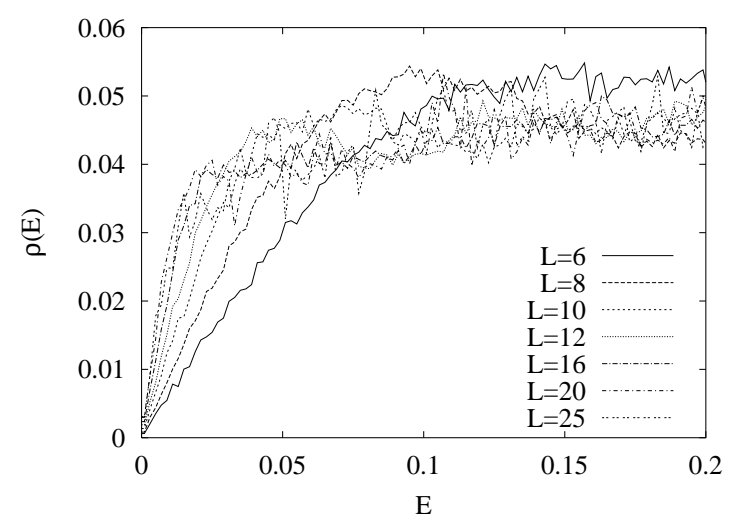

Fig. 3. Density of states for $d=0.1$, disorder strength $W=9$, gap $\Delta=1$, and various system sizes $L$.

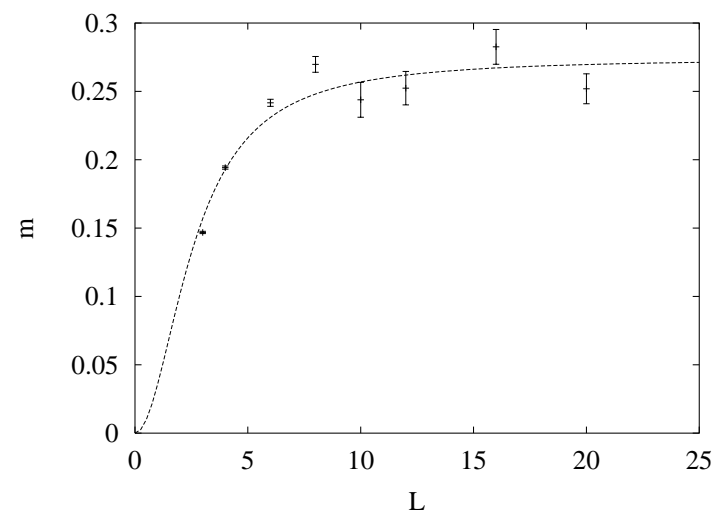

Fig. 4. Slope $m$ of the microgap for correlation length $d=0.1$, disorder strength $W=18$, and gap $\Delta=1$ as a function of system size $L$. The dashed line is a fit of the function in Eq. (3) with $m_{\infty}=0.27$ and $\xi=2.6$.

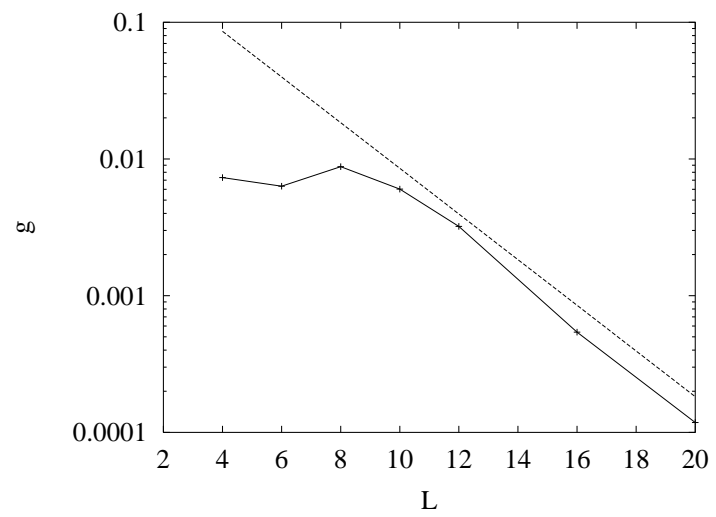

Fig. 5. Semi-logarithmic plot of the Thouless numbers $g$ near zero energy for correlation length $d=0.1$, disorder strength $W=18$ and gap $\Delta=1$ as a function of system size $L$. The dashed line corresponds to an exponential decrease with localization length $\xi=2.6$.

Fig. 4 showing good agreement.

To summarize, we have presented evidence that in disordered $d$-wave superconductors details of disorder are relevant. For long-ranged disorder, we find the expected power law behavior of the quasi-particle density of states. For short-ranged disorder, we have shown that DoS contains information about the localization properties of the system in contrast to conventional disordered system. In particular, we could extract the localization length from the system size dependence of the microgap in the DoS. Calculations of the Thouless conductance of the system corroborate our interpretation.

1) P. W. Anderson: Phys. Rev. 109 (1958) 1492.

2) A. Altland and M. R. Zirnbauer: Phys. Rev. B 55 (1997) 1142

3) for details, see B. Huckestein and A. Altland: (2000) condmat/0007413; A. Altland, B. D. Simons, and M. R. Zirnbauer: Phys. Rep. 359 (2002) 283.

4) A. W. W. Ludwig, M. P. A. Fisher, R. Shankar, and G. Grinstein: PRB 50, (1994) 7526.

5) A. Nersesyan, A. Tsvelik, and F. Wenger: Phys. Rev. Lett. 72 (1994) 2628.

6) V. Gurarie, (1999) cond-mat/9907502. 